\title{
ON THE SPECTROPHOTOMETRIC IDENTIFICATION OF LATE TYPE BINARIES IN OPEN CLUSTERS
}

\author{
C. Bettis and D. Branch \\ Dept. of Physics and Astronomy \\ The University of Oklahoma \\ (Presented by O. G. Franz)
}

\begin{abstract}
RESUMEN
Usando tanto los valores medidos como modelos atmosféricos, se han calculado los anchos equivalentes de las líneas $\mathrm{D}$ del $\mathrm{Na}$ para estrellas binarias hipotéticas de tipo tardío de la secuencia principal. Es posible un aumento en el brillo de las líneas $\mathrm{D}$ de un $90 \%$ sobre el valor para una estrella sola, cuyo color $\mathbf{B}-\mathrm{V}$ es el mismo que del par combinado. Se sugiere que este efecto puede usarse para identificar binarias en cúmulos galácticos, en particular para aquellas binarias cuyas componentes difieren en brillo por más de 3 mag.
\end{abstract}

\begin{abstract}
ABSTRAC'T
Using both measured values and model atmospheres, the equivalent widths of the Na $D$ lines of hypothetical late type main sequence binaries are calculated. Enhancements in the $\mathrm{D}$ lines of $90 \%$ over the value for a single star whose $\mathrm{B}-\mathrm{V}$ color is the same as the composite $B-V$ are possible. It is suggested that this effect may be used to identify binaries in galactic clusters, in particular those binaries whose components differ by more than 3 mag in brightness.
\end{abstract}

\section{INTRODUCTION}

The selection effects involved in identifying binary stars are too well known to go into here. It is sufficient to point out that it woud be of great interest to find ways of identifying binaries that did not depend on their orbital parameters or distance moduli. We have developed two such methods and hope that they will be useful in identifying binaries in at least galactic clusters. The first which we have already applied to the Hyades, Praesepe and the Pleiades (Bettis 1975) involves 3-color photometry, the fact that a binary is brighter than a single star of the same color and same elementary statistics. This technique is good for binaries whose components differ by less than about 3 mag. The second method, reported on here, is a spectrophotometric one making use of the properties of the $\mathrm{Na} \mathrm{D}$ lines in solar and later type stars; it is hoped that it will complement the first.

\section{THE Na D LINES}

Given the number of equivalent width measurements in the literature, it is possible to calculate the strengths of $\mathrm{Na} \mathrm{D}$ lines in composites without resorting to model atmospheres. $\mathrm{B}-\mathrm{V}$ colors and equivalent widths of composites were calculated using (1) a mean relation between absolute visual magnitude and $\mathrm{B}-\mathrm{V}$ for nearby main sequence stars (Woolley et al. 1970), (2) a mean relation between the equivalent width of the $D_{1}+D_{2}$ doublet and $B-V$ color (Figure 1) constructed from the data given by Tinsley (1967) and Spinrad (1962), and (3) a relation between $M_{v}$ and the monochromatic flux at $5900 \AA$, estimated from the absolute measurements of Whiteoak (1967). The composite equivalent widths were obtained by weighting the equivalent widths of the component stars according to the $5900 \AA$ flux.

The composite equivalent widths, also shown in Figure 1, are in general significantly greater than 


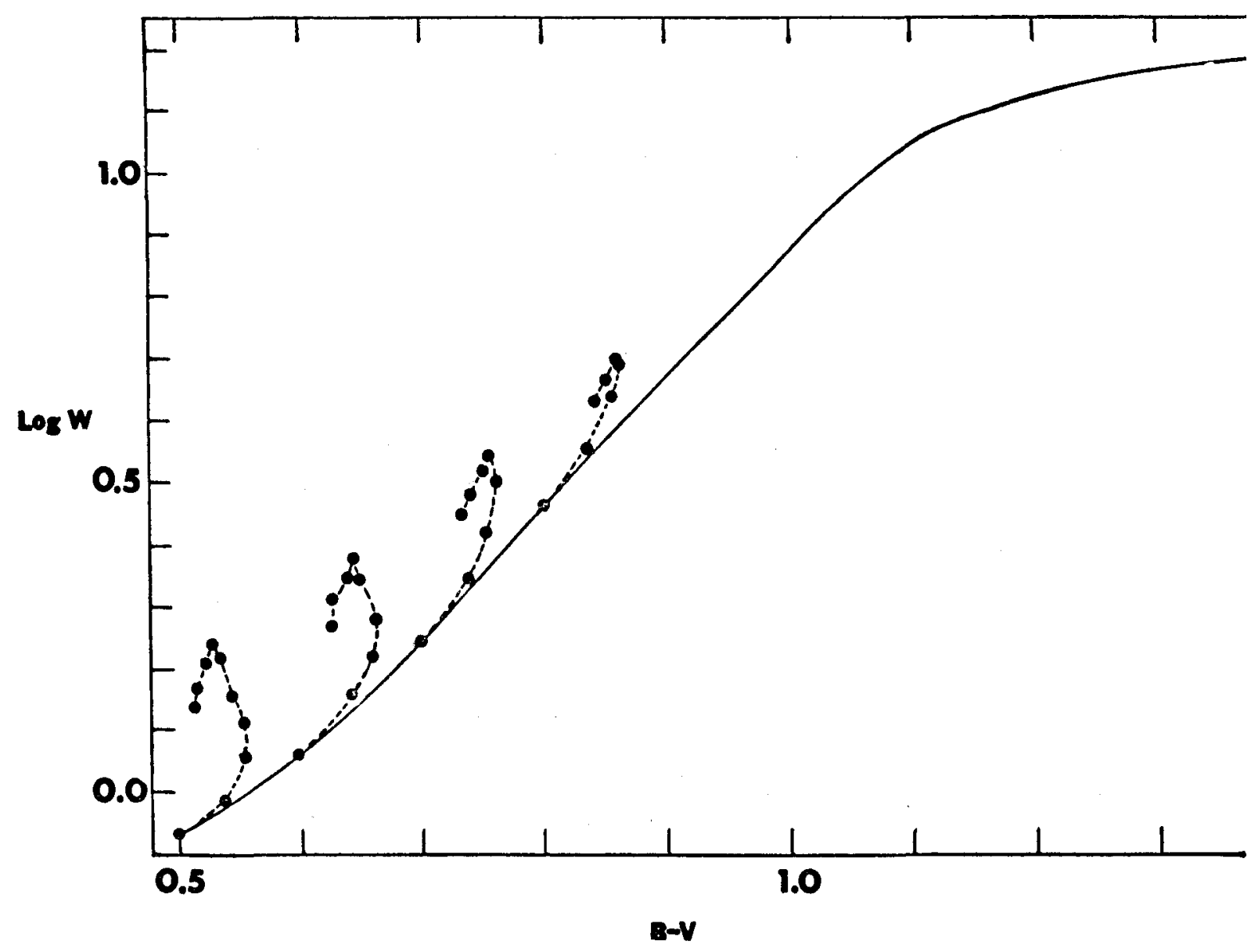

Fio. 1. Mean relation between $\mathrm{Na} \mathrm{D}_{1}+\mathrm{D}_{2}$ equivalent widths and $\mathrm{B}-\mathrm{V}$ color (full line). Filled circles represent equivalent widths and colors of composites consisting of a primary having $\mathrm{B}-\mathrm{V}=0.5,0.6,0.7$ or 0.8 and secondaries having $\mathrm{B}-\mathrm{V}=1.4$.

those of single stars of the same color. In the most extreme case, the pair of stars having $\mathrm{B}-\mathrm{V}=0.5$ and 1.1 forms a composite which has an equivalent width $90 \%$ greater than the single star having the same color, $\mathrm{B}-\mathrm{V}=0.53$. A star having $\mathrm{B}-\mathrm{V}=0.5$ cambined with any star having $B-V$ in the range 0.8 to 1.4 , forms a composite which has $\mathrm{D}$ lines enhanced by more than $30 \%$. Judging from Figure 1, stars redder than $\mathrm{B}-\mathrm{V}=1.4$ would also produce significant effects, but the problem would become complicated by $\mathrm{TiO}$ contamination of the $\mathrm{D}$ lines in $M$ stars. It must be mentioned that the relation between equivalent width and color is not well determined for $\mathrm{B}-\mathrm{V}>1.0$. If the adopted curve is too high, the effects of faint companions will be over-estimated. However, a theoretical relation based on model atmosphere calculations of $\mathrm{D}$-line equivalent widths (Cayrel de Strobel 1969) implies the opposite, i.e. that the effects of duplicity have been underestimated.

The actual effects of duplicity on equivalent widths measured on microphotometer tracings might be somewhat less drastic than implied by Figure 1 . In the most extreme cases of D-line enhancement the major contribution of the cooler star is to produce broad, shallow wings on the composite profile (see Figure 2, based on line profiles calculated from the model atmospheres of Carbon and Gingerich 1969); for each composite a red color index, based on the moncchromatic fluxes of the 


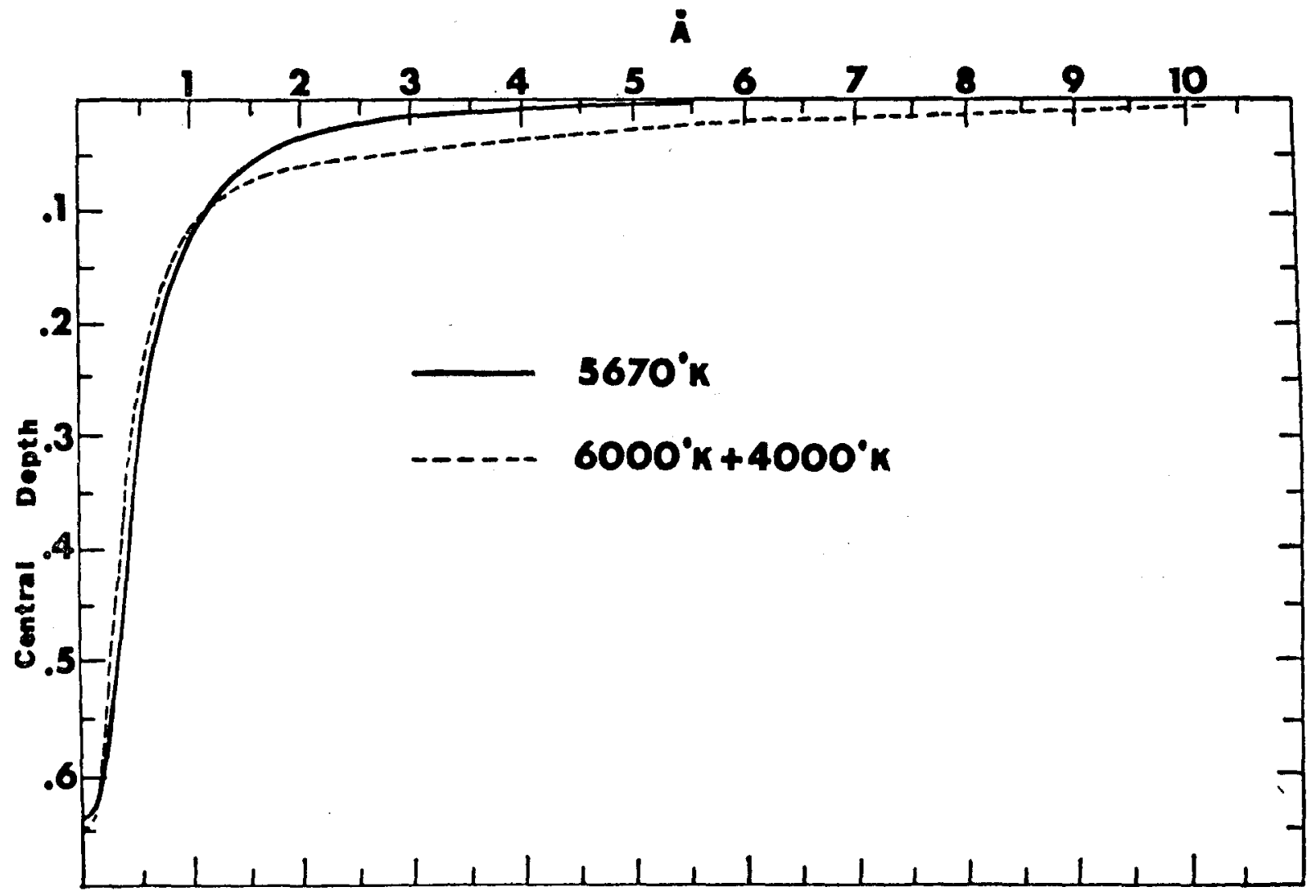

Fro. 2. Calculated profiles of the $\mathrm{Na} \mathrm{D}_{2}$ line, based on model atmospheres having $\log \mathrm{g}=4.0$.

models at $6095 \AA$ and $6994 \AA$, was computed. A single model having the same red color was then obtained by interpolation in the Carbon-Gingerich grid. The unexpectedly shallow wings might go unnoticed, to some extent, by the measurer.

The effects of duplicity on narrow-band photoelectric measurements of the D-lines might be quite serious, since such measurements take full account of any line wings, however shallow, which fall within the passbands. This suggests the interesting possibility of spectrophotometric detection of binary stars in clusters, where chemical composition differences are presumably absent. Such a method would offer the very attractive opportunity to detect relatively faint companions. With this possibility in mind we have examined existing photoelectric Dline indices for Hyades dwarfs. A plot of Price's (1966) D-line index against $B-V$ definitely does contain significantly more scatter than expected on the basis of his quoted observational uncertainties; it is quite possible that the extra scatter is caused by duplicity. We have been unable to reach any conclusion regarding Taylor's (1970) index because of the few measured Hyades per unit color interval.

\section{CONCLUSION}

One of us (C.B.) intends to observe the D-lines of late type Hyades. By knowing the observational error and assuming a fixed chemical composition, we hope to be able to detect with confidence such binaries as described above by noting where they fall on a plot of $\mathrm{B}-\mathrm{V}$ vs. equivalent width.

It may be possible to extend this approach to field stars by finding a set of features on which 
the effects of duplicity would be such that they would be very unlikely to be confused with age or abundance effects.

\section{REFERENCES}

Bettis, C. 1975, Pub. A.S.P., 87, 707.

Carbon, D. F., and Gingerich, O. 1969, in Theory and Observation of Normal Stellar Atmospheres, ed. $O$. Gingerich (Cambridge, Mass.: The MIT Press), 377.
Cayrel de Strobel, G. 1969, in Theory and Observation of Normal Stellar Atmospheres, ed. O. Gingerich (Cambridge, Mass.: The MIT Press), 35.

Price, M. J. 1966, M.N.R.A.S., 134, 135.

Spinrad, H. 1962, Ap. J., 135, 715.

Taylor, B. J. 1970, Ap. J. Suppl., 186, 177.

Tinsley, B. 1967, Pub. Dept. Astr. Univ. Texas, Series II, 1, 15.

Whiteoak, J. B. 1967, Ap. J., 150, 521.

Woolley, R., Epps, E. A., Penston, M. J., and Pocock, S. B. 1970, Roy Obs. Ann., No. 5.

\section{DISCUSSION}

Abt: Mr. Bettis has in press a paper which shows that with careful photometry of open cluster members one can predict with fairly good success the stars that are double because they tend to fall high on the main sequence band. This present paper describes another technique for selecting binaries. It seems very reasonable, although $I$ would like to see the detailed calculations before believing that a full 3-mag difference in brightness would be observable. 